\title{
Immunonutrition and surgical practice
}

\author{
Leán O'Flaherty* and David J. Bouchier-Hayes \\ Department of Surgery, Royal College of Surgeons in Ireland, Beaumont Hospital, Dublin 9, Republic of Ireland
}

\begin{abstract}
Immunonutrition generally refers to the effect of the provision of specific nutrients on the immune system. These nutrients typically have immunoenhancing properties, and recent advances in nutrition support involve studies designed to exploit the desirable biological properties of these nutrients. The term immunonutrition strictly implies that we are focusing on the effect of certain nutrients on aspects of the immune system. However, in reality immunonutrition also refers to studies that not only examine the function of lymphocytes and leucocytes, but which also study the influence of key nutrients on the acute-phase response, the inflammatory response and on gastrointestinal structure and function. The interest, therefore, is on the impact of immunonutrition on all aspects of host defence mechanisms in response to a catabolic stress. Major surgery evokes an acute-phase response, a transient immunosuppression and alterations in gastrointestinal function. Normal function is usually restored after a few days; however, in a subgroup of patients homeostasis may be lost and development of the systemic inflammatory response syndrome (SIRS) ensues. Results of recent clinical trials suggest that provision of immunomodulatory nutrients, including glutamine, arginine, $n-3$ polyunsaturated fatty acids and dietary nucleotides, may promote restoration of normal tissue function post-operatively and prevent the occurrence of SIRS.
\end{abstract}

Immunology: Glutamine: Arginine: Taurine

\section{Host response to surgery}

\section{The acute-phase response}

In response to a tissue injury the host immediately mounts an inflammatory response designed to initiate wound healing and combat infection, which is referred to as the acute-phase response. This response centres around the activation of the circulating monocyte, which immediately releases tumour necrosis factor alpha (TNF- $\alpha$ ), followed closely by interleukins (IL) 1 and 6 . These cytokines are the primary mediators of the inflammatory response, and orchestrate the complex series of events that follow monocyte activation (Giroir, 1993; Baumann \& Gauldie, 1994; Bone, 1996; Davies \& O’Hagen, 1997; Chang \& Bistrian, 1998).

The main target of primary cytokines appears to be the vessel wall, which is ideally located to control the inflammatory response. Endothelial cells are stimulated to produce a wave of secondary inflammatory mediators, including reactive oxygen intermediates, prostaglandins and leukotrienes. Release of prostaglandins immediately affects vascular tone and results in increased vascular permeability, designed to increase $\mathrm{O}_{2}$ and nutrient delivery to the injured tissue.

Chemokines such as IL- 8 are also generated and attract leucocytes to the injured tissue. Increased expression of adhesion molecules such as endothelial leucocyte adhesion molecule 1 and intracellular adhesion molecule 1 on the endothelium is also involved in recruitment of leucocytes which are employed to fight infection and restore normal tissue function.

The liver is another major target of the primary inflammatory mediators, where they induce the hepatic acute-phase response. Essential metabolites necessary to cope with critical illness, such as C-reactive protein, are produced by the liver at the expense of proteins such as albumin. The production of these acute-phase proteins is synergistically enhanced by glucocorticoids and inhibited by insulin and growth factors.

Primary cytokines also have profound metabolic consequences. Collectively, they cause generalized fever, increased $\mathrm{O}_{2}$ consumption and increased metabolism of fats, glucose and proteins. Further alterations include induction of the complement and coagulation cascades, an increased 
temperature set point in the hypothalamus, tachycardia, anorexia and pain. These effects are thought to be mediated by prostaglandins. The acute-phase response generally lasts 24-48 h. Resolution of the acute-phase response is achieved with the help of circulating anti-inflammatory cytokines such as IL-4 and IL-10, and circulating cytokine antagonists including IL-1 receptor antagonist.

\section{Immunodepression}

Neutrophil function after surgery has been shown to be impaired within $24 \mathrm{~h}$. Chemotaxis, respiratory burst and phagocytosis are all affected (Utoh et al. 1988). General anaesthesia and surgery also depress monocyte or macrophage activity. The effect of surgery has been attributed to a reduced trans-hepatic portal blood flow, which normally stimulates macrophage phagocytosis. Blood transfusions administered during the operation can also depress monocyte activities; however, these effects are not usually seen until post-operative day 7. Monocyte function is also impaired with the widespread use of antibiotics (Athlin et al. 1991).

Major surgery may have a profound effect on the number of total T-cells, pan T-cells, suppressor or cytotoxic T-cells and natural killer cells, which are all reduced at postoperative day 3 (Slade et al. 1975). These impairments are usually transient, and it is imperative that normal function is restored quickly in order to facilitate wound healing and to combat infection.

\section{Gastrointestinal function following major surgery}

Many events characteristic of surgery and stress can adversely affect the gastrointestinal tract. Fasting for tests, obstructive malignancies and chemotherapy can all result in a failure to supply adequate nutrition. Lack of nutrition has several immediate pathophysiological consequences, including inhibition of saliva and digestive tract secretions, reduced gastrointestinal motility and splanchnic circulation, absence of stimulatory nutrients such as short-chain fatty acids which can upset the normal gastrointestinal flora, atrophy of the mucosa of the small intestine and colon, and impaired gastrointestinal-associated lymphoid tissue function. An increase in the concentration of pathogenic organisms in the gastrointestinal lumen and a reduction in the number of probiotic bacteria may precede the passage of pathogenic organisms from the gastrointestinal lumen to the bloodstream, termed gastrointestinal-associated bacterial translocation. The gastrointestinal tract has also been implicated in the pathogenesis of sepsis, by allowing bacterial translocation, and three main events are prerequisites for bacterial translocation to occur: loss of gastrointestinal barrier function; alteration in gastrointestinal flora; derangements in host immune defence mechanisms (Deitch, 1994). Any trauma involving ischaemic injury, haemorrhage or severe shock may result in mucosal injury and consequent loss of gastrointestinal integrity. This process may aggravate the incidence of bacterial translocation. Furthermore, major surgery can reduce the splanchnic blood flow, limiting the exposure to stimulatory hormones and depriving the enterocytes of $\mathrm{O}_{2}$. Peristalsis, particularly in the stomach, is a transient side-effect of major surgery, and may also have an adverse effect on gastrointestinal function.

The cumulative effect of these insults on the gastrointestinal tract is impaired nutrient absorption and reduced gastrointestinal integrity. Impaired amino acid absorption has received attention in recent years, after it was discovered that certain amino acids are conditionally essential for the gastrointestinal tract (Gardiner \& Barbul, 1993; Souba, 1993; Gardiner et al. 1995).

\section{The systemic inflammatory response syndrome}

In some patients, the acute phase fails to resolve when homeostasis is lost. Sepsis syndrome can develop where there is an exaggerated pro-inflammatory response and where pro-inflammatory cytokines, particularly TNF- $\alpha$ and IL-6, persist in the bloodstream. Bacterial translocation may also play a role. Multi-organ dysfunction can develop where systemic pro-inflammatory cytokines spill over into end organs such as the lung, liver and kidneys, where they can cause irreparable damage.

While endless research has been carried out into the development of systemic inflammatory response syndrome (SIRS), this outcome is only one of two possible sequelae which may be manifested as a result of loss of homeostasis. The other possible outcome has been termed compensatory anti-inflammatory response syndrome, where immunodepression is the predominant state (Davies \& O'Hagen, 1997). In the past, treatment of SIRS has focused on the nidus of infection, inhibition of lipopolysaccharide, or blocking the production of toxic cytokines.

Treatment of specific infections relies on antibiotic therapy, which is ineffective in the treatment of SIRS, as antibiotics are unable to reduce the synthesis or toxicity of the endogenous mediators involved (Giroir, 1993). Antilipopolysaccharide strategies have also met with little success. Antibodies directed against the O-polysaccharide component have met with disappointing results, as this component is structurally different for each Gram-negative bacterium (Hoffman \& Natanson, 1993). Therapies directed against cytokine production have targeted those cytokines which are the primary mediators of SIRS, i.e. TNF- $\alpha$, IL-1 and IL-6. An agent designed to block cytokine production must do so at a transcriptional or translational level, as these mediators are produced from de novo synthesis during SIRS and are not pre-formed. Anti-TNF- $\alpha$ has met with disappointing results in human trials, whereas anti-IL-1 has conferred some benefits if given within $1-2 \mathrm{~d}$ to those patients with a greater than $24 \%$ risk of death (Davies \& O'Hagen, 1997). The main difficulty with these treatments is that they need to be given pre-emptively. By the time a patient is seriously ill multiple cytokines have been produced and many monocyte populations have been activated. This situation may be the reason why so many single agent therapies are unsuccessful. Furthermore, the main treatment options target pro-inflammatory mediators, and neglect the fact that immunosupression or inadequate anti-inflammatory mediators may also be contributing to the loss of homeostasis. 


\section{Immunonutrition}

An ideal therapy would be one that has anti-inflammatory properties to promote a natural resolution of the acute-phase reaction, and which also has immunostimulatory and gastrointestinal trophic effects. Thus, nutritional intervention has been implicated, specifically nutritional pharmacology or immunonutrition.

The more prominent of these immunoenhancing dietary agents are glutamine, arginine, $n-3$ polyunsaturated fatty acids and dietary nucleotides. Recent studies suggest that taurine may also play a role.

\section{Glutamine}

Glutamine is one of the most abundant amino acids in the body (Bergstrom et al. 1974), and is noted for its role in nucleotide biosynthesis, as a $\mathrm{N}$ courier between cells and in acid-base balance. There is convincing evidence that glutamine plays a vital role in the immune response, as it is rapidly absorbed by immune cells for fuel, and promotes proliferation, cytokine release and tumour cell cytotoxicity. It is also required for production of the antioxidant GSH (Hong et al. 1990, 1992), and is believed to be an essential fuel for the gastrointestinal tract (Lacey \& Wilmore, 1990; Hall et al. 1996).

The potential benefits of glutamine supplementation may be related to the fact that it is a conditionally-essential nutrient required during catabolic responses to ensure optimal tissue function. This property separates it from other immunonutrients which confer benefits through their pharmacological ability to upregulate the immune response as opposed to correcting a deficiency state.

Catabolic states induce a relative deficiency of glutamine. Stress hormones such as glucocorticoids evoke an outpouring of glutamine from skeletal muscle in order to maintain plasma levels. Plasma glutamine is specifically taken up by the gastrointestinal tract, liver, and kidney, and requirements may increase during stress, eventually leading to a reduced plasma pool (Souba, 1993). It has been claimed that immune function should be intact at a plasma glutamine level of $600 \mu \mathrm{mol} / 1$ or higher. Deficiency levels of $400 \mu \mathrm{mol} / \mathrm{l}$, on the other hand, may jeopardize immune defences (Wilmore \& Shabert, 1998). These low levels have been observed in trauma patients, burn patients and during chemotherapy. Post-operative levels cited in the literature remain above $600 \mu \mathrm{mol} / 1$, indicative of a functioning immune system. The actions of glutamine on host defences are multi-faceted, and it is possible that glutamine is required post-operatively specifically for its gastrointestinal trophic effects. This requirement may have a significant impact on choice of route of feeding, parenteral or enteral.

It has been argued that parenteral supplementation of glutamine is preferable over enteral supplementation, as it corrects plasma glutamine levels more efficiently (Lacey \& Wilmore, 1990; Fish et al. 1997). Of the enteral glutamine, $50-70 \%$ is consumed by the gastrointestinal tract and liver, and subsequently less is available to elevate blood and muscle concentrations. However, this argument overlooks the benefits of oral glutamine on gastrointestinal structure and function.

The earliest glutamine supplementation studies involved total parenteral nutrition (TPN), and therefore bypassed the gastrointestinal tract. Early studies encountered problems with parenteral supplementation due to the instability of glutamine in solution; however, this problem has been overcome by the development of stable glutamine dipeptides, where glutamine is bound to alanine or glycine. The dipeptide is rapidly hydrolysed to the free amino acid form after intravenous infusion (Furst \& Stehle, 1993).

The first major clinical studies to examine the benefit of glutamine supplementation via TPN were carried out on adult bone-marrow transplant patients. Adult bone-marrow transplant patients were randomized to receive glutaminesupplemented TPN $24 \mathrm{~h}$ after surgery, and fewer clinical infections were documented in the supplemented group who had a reduced incidence of microbial colonization (McBurney et al. 1994). Average length of hospital stay was reduced from 36 to $29 \mathrm{~d}$. In a separate publication (Ziegler et al. 1998), the same group reported that the benefits in this population may be related to an increased circulation of lymphocytes, both total lymphocytes and $\mathrm{T}$ lymphocyte subsets.

It has been estimated that a typical post-operative patient requires $0.3 \mathrm{~g}$ glutamine $/ \mathrm{kg}$ per d, equivalent to $20 \mathrm{~g}$ in a $60-70 \mathrm{~kg}$ individual. In more stressful situations, such as trauma or burns, up to $40 \mathrm{~g} / \mathrm{d}$ may be necessary to correct the deficit (Furst \& Stehle, 1993; Hall et al. 1996).

Griffiths et al. (1997) randomized eighty-four criticallyill patients with multi-organ failure to receive either glutamine-supplemented or standard TPN. After 6 months, survival was significantly greater in the supplemented group (twenty-four of forty-two $v$. fourteen of forty-two; $P=0 \cdot 049)$. Houdijk et al. (1998) randomized sixty trauma patients to either a standard enteral feed or one containing $14.2 \mathrm{~g}$ glutamine/l within $48 \mathrm{~h}$ of admission. There was a significantly reduced rate of infection in the supplemented group, with $17 \%$ v. $45 \% \quad(P<0.02)$ presenting with pneumonia, and bacteraemia being detected in just $7 \% v$. $42 \%(P<0.005)$. There was also a significant difference in the number of patients developing sepsis (one in the supplemented group $v$. eight in the control group; $P<0.02)$. Finally, this study demonstrated that enteral supplementation could indeed maintain the plasma pool; plasma glutamine levels were significantly higher $(P<0.05)$ in the supplemented group on post-operative days 3,4 and 5 .

There has been surprisingly little published on glutamine supplementation in routine post-operative patients. In a parenteral supplementation study, post-operative colon cancer patients were given 0.3 g glutamine $/ \mathrm{kg}$ per d parenterally for a total of $5 \mathrm{~d}$. In the group that received supplementation an improved $\mathrm{N}$ balance was observed, lymphocyte counts were improved and hospital stay was significantly reduced by $6.2 \mathrm{~d}(P<0.05$; Morlion et al. 1998). O'Riordán et al. (1996) administered glutamine-enriched TPN to postoperative patients with colo-rectal cancer and documented enhanced T-cell mitogenic responses. 


\section{Arginine, n-3 polyunsaturated fatty acids and dietary nucleotides}

Arginine has been shown to be immunostimulatory by increasing thymic size, by promoting T-cell proliferation, and macrophage and natural killer cell function (Barbul, 1986; Barbul et al. 1990; Reynolds et al. 1998). Two key roles of arginine are believed to be responsible for its immunoenhancing properties: its role as a precursor for both polyamine and NO synthesis (Evoy et al. 1998). Polyamines play a key role in DNA synthesis and the regulation of the cell cycle, while NO is involved in many physiological events, including maintenance of vascular tone, coagulation, regulation of the immune system and of gastrointestinal function.

The majority of studies have looked at arginine in conjunction with $n-3$ polyunsaturated fatty acids and dietary nucleotides (IMPACT; Sandoz Nutrition, Bern, Switzerland). n-3 Polyunsaturated fatty acids exert both anti-inflammatory properties by down regulating prostaglandin $\mathrm{E}_{2}$ and pro-inflammatory cytokines, and immunostimulatory effects by enhancing T-cell and natural killer cell activity (Alexander et al. 1986; Gottschlich et al. 1990). The $n-3$ polyunsaturated fatty acids eicosapentaenoic acid and docosahexaenoic acid are more readily converted to active forms than $\alpha$-linolenic acid and, therefore, may have more potent effects. Dietary nucleotides promote protein synthesis in mammalian tissues and are also believed to be involved in T-cell functions.

There have been numerous studies of the clinical, immunological, nutritional and biochemical effects of IMPACT. Senkal et al. (1997) randomized 154 patients with upper gastrointestinal cancer to receive IMPACT or a standard enteral formula post-operatively. They observed a non-significant reduction in early complications (within the first $5 \mathrm{~d}$ ), and a significant reduction in late complications ( $n 5$ v. $n 13 ; P<0.05$ ). Previously, the same group reported on a smaller study of forty-four cancer patients (Senkal et al. 1995). In this study IMPACT down regulated TNF- $\alpha$ and IL-6, and stimulated lymphocyte immune function. Daly et al. (1992) have also examined IMPACT in post-operative cancer patients and observed an improved $\mathrm{N}$ balance, enhanced lymphocyte activity and lymphocyte proliferation. These benefits were clinically confirmed by a significant reduction in infection rates and a reduction in wound healing complications, while average length of admission fell from $20 \cdot 2$ to $15 \cdot 8 \mathrm{~d}$.

Braga et al. (1996b) randomized seventy-seven cancer patients to receive either arginine-supplemented TPN, IMPACT or a standard enteral feed post-operatively. In the IMPACT group there was a faster recovery of phagocytosis, delayed hypersensitivity response, pre-albumin and retinolbinding protein. Clinically, this group had a significantly shorter length of hospital stay, a lower sepsis score and a trend to decreased infection. Daly et al. (1995) compared IMPACT with Traumacal (Bristol-Meyers Squibb, Evansville, IN, USA) in sixty post-operative cancer patients, and as before they observed benefits in the IMPACT group. Complication rates and length of hospital stay were reduced, and this reduction was correlated at a cellular level with a down regulation in prostaglandin $\mathrm{E}_{2}$ release. In a second limb to the study half the patients continued with their nutritional regimen via overnight feeding after discharge. The rehospitalization rate was reduced in the overnightfeeding group compared with those patients sent home on a normal diet, regardless of whether they were receiving IMPACT or Traumacal.

Braga et al. (1996a) conducted the only study to examine peri-operative IMPACT administration with standard enteral nutrition in forty patients with colo-rectal cancer. They reported an increase in phagocytosis and a reduced C-reactive protein release in the IMPACT group. This study was the only one to examine the effect of IMPACT on gastrointestinal metabolism more closely. They found IMPACT to increase intestinal microperfusion, $\mathrm{O}_{2}$ metabolism and NO levels in the gastrointestinal tract.

Results from a large multi-centre trial were published by Bower et al. (1995). The study involved 296 patients in the intensive treatment unit. While there was a trend towards a reduced length of stay and infection rates in the IMPACT group as a whole, the most significant results were observed in the subgroup identified as having sepsis. Length of hospital stay in this group was reduced by $10 \mathrm{~d}$ and the incidence of nosocomial infections fell by $60 \%$. On further analysis it was revealed that the IMPACT sepsis group who received their full nutritional prescription fared best, with length of stay reduced by $11.5 \mathrm{~d}$. There were no differences noted in nutritional variables or mortality rates among any of the groups.

More recently, Atkinson et al. (1998) studied 398 patients in the intensive care unit who were randomized to receive either a control feed or IMPACT within $48 \mathrm{~h}$ of admission. The best results were achieved in those patients who tolerated early enteral nutrition. The number of days on mechanical ventilation was reduced from 10.5 to 6 and length of hospital stay was $20 \mathrm{~d} v .15 \cdot 5 \mathrm{~d}$.

In a smaller study of thirty-two patients with multi-organ failure, fewer 'SIRS' days per patient, an improved multiorgan failure score and lower circulating C-reactive protein levels were reported (Weimann et al. 1998). However, there was no difference in IL-2 receptor, mortality, length of hospital stay or infection rates.

There has been concern raised about the safety of arginine when it is administered to cancer patients, as arginine has been shown to enhance the proliferation of certain tumour types. Arginine is required for synthesis of polyamines, which are in turn regulators of cell growth, and in some tumour types arginine is essential for cell growth. Park et al. (1992) demonstrated an increase in tumour proliferation markers in patients with breast cancer given arginine supplements. Arginine has been shown to modulate the growth of breast cancer cell lines both in vitro and in vivo by NO-dependent and -independent means (Edwards et al. 1997). An arginine-depleted diet inhibited growth of colon tumour cells in a murine model in a study by Yeatman et al. (1991). This finding was attributed to a requirement for arginine for growth by this particular cell type. Conversely, arginine has been shown to potentiate IL-2 antitumour immunotherapy (Lieberman et al. 1992).

While it has been argued that these concerns may not be relevant to post-operative cancer patients after the tumour has been excised, it is clear that extra work is urgently 
needed to fully clarify the safety issues related to arginine supplementation in cancer patients.

\section{Taurine}

Taurine is one of the most abundant amino acids in many cell types, where its roles include membrane stabilization, osmoregulation and $\mathrm{Ca}$ flux regulation (Gaull \& Rassin, 1979; Huxtable, 1992). Interest in taurine as an immunomodulator was generated by the discovery of its antioxidant capacity and its ability to prime leucocytes and to regulate the release of pro-inflammatory cytokines (Gordon et al. 1986; Masuda et al. 1986; McLoughlin et al. 1991; Watson et al. 1994).

Evidence suggests that taurine may be a conditionallyessential amino acid. Intestinal absorption has been shown to be reduced under stressful conditions in vitro (O'Flaherty et al. 1997). Gastrointestinal intracellular taurine has been shown also to be depleted in multi-trauma patients and elective cholecystectomy patients (Ahlman et al. 1995a,b). We have shown that supplemental taurine given to stressed intestinal cells in vitro can maintain absorption rates, promote the enterocyte cell cycle and prevent stress-induced apoptosis or cell death, which clearly indicates a gastrointestinal trophic effect (L O'Flaherty, PP Stapleton, HP Redmond and D Bouchier-Hayes, unpublished results).

Taurine has also been shown to have immunomodulatory effects. In a murine model of sepsis taurine supplementation conferred immune benefits by down regulating TNF- $\alpha$ release and upregulating anti-bacterial capacity, as assessed by peritoneal macrophage superoxide generation (O'Flaherty et al. 1998). In the neutrophil taurine is a potent modulator of myeloperoxidase ( $E C$ 1.11.1.7), a key enzyme in the respiratory burst chain (Stapleton et al. 1998). This action enables it to sequester the potent reactive oxygen intermediate, $\mathrm{HOCl}$, rendering it unable to induce tissue damage. In the lymphocyte, addition of taurine to the culture medium has been shown to promote proliferation and protect against oxidant-induced damage (Pasantes-Morales et al. 1985).

We have recently completed a clinical trial involving seventeen elderly elective surgery patients. This trial was a randomized placebo-controlled study, approved by the local Ethics Committee at Beaumont Hospital, Dublin, comparing a standard enteral feed with a taurinesupplemented feed $(1 \mathrm{mg} / \mathrm{ml})$ in the peri-operative period. Feeding was commenced $2 \mathrm{~d}$ before surgery, and continued until post-operative day 5. Mortality rates, length of hospital stay and routine biochemical variables were similar between the two groups. However, taurine supplementation appeared to modulate the post-operative cytokine profile. Two key cytokines were regulated by taurine. The pro-inflammatory cytokine IL- $1 \beta$ was significantly reduced on post-operative days 1,3 and $5(P=0 \cdot 049)$. Conversely, the anti-inflammatory cytokine IL-10 was enhanced on post-operative days 1 and 3 . It could be speculated that the net effect would be a beneficial one for the patient. By down regulating a critical pro-inflammatory cytokine and up regulating an anti-inflammatory cytokine, a more efficient resolution of the acute-phase response would be encouraged. These benefits may be related to the provision of taurine during the acute-phase response. Indeed, both the plasma and serum levels of taurine were higher in the supplemental group in the early post-operative period (L O'Flaherty, PP Stapleton, HP Redmond and D BouchierHayes, unpublished results).

\section{Phospholipids, soluble fibre and probiotic bacteria}

The list of possible immunonutrients is ever expanding. Many of the components of cellular membranes grouped together as phospholipids may also have immunostimulatory effects. Phosphatidylcholine and phosphatidylinositol have been shown to reduce bacterial translocation in vivo after $90 \%$ liver resection (Wang et al. 1994). Gangliosides may have potent effects on cellular immune reactions (Yamaguchi et al. 1997). The health benefits of fibre are by now well established. Soluble fibre has been particularly studied in relation to its cholesterol-lowering capacity, and it has recently been exploited for its gastrointestinal trophic effects. In particular, pectin has been shown to stimulate gastrointestinal-associated lymphoid tissue (Zaporozhets et al. 1991) and to protect the gastrointestinal mucosa against oxidative damage (Kohn \& Keithly, 1989). The benefits of oat fibre and guar gum are described as being similar to those of pectin. Finally, probiotic bacteria such as Lactobacillus plantarum may be employed to prevent colonization of the gastrointestinal tract by pathogenic organisms (Bengmark, 1998). Currently, a nutrition formula is being developed which contains L. plantarum-fermented oat fibre. Development of such formulas will pave the way for immunonutrition research to expand into new and exciting areas.

\section{Conclusion}

It would appear that provision of immunostimulatory and conditionally-essential nutrients during the early postoperative period can effectively promote human immune defence mechanisms and encourage an efficient resolution of the acute-phase response. These benefits are derived through the promotion of gastrointestinal function and regulation of cytokine responses, and by enhancing immune function. It is apparent that there is no one specific nutrient that possesses all these qualities, and formulas containing a number of immunonutrients seem to have more potent effects. It would also appear that the most dramatic effects have been observed in those patients who are critically ill, have developed sepsis, or in those patients who have received their full nutritional prescription. Studies are warranted to develop pre-operative screening methods to identify those patients at risk of developing sepsis. These patients may benefit most from a 'cocktail' immunonutrient formula. Until such a screening protocol is established, provision of such a formula to all patients may be undertaken as a prophylactic measure. This formula should ideally be given peri-operatively or early in the post-operative period, as it has been observed that proinflammatory cytokines are present in the circulation within hours of the initial surgical incision. Homeostasis may already have been lost in susceptible patients if initiation of feeding is delayed. 


\section{References}

Ahlman B, Ljungqvist O, Andersson K \& Wernerman J (1995a) Free amino acids in the human intestinal mucosa; impact of surgery and critical illness. Clinical Nutrition 14, 54-55.

Ahlman B, Ljungqvist O, Persson B, Bindsley L \& Wernerman J (1995b) Intestinal amino acid content in critically ill patients. Journal of Parenteral and Enteral Nutrition 19, 272-278.

Alexander JW, Saito H \& Trocki O (1986) The importance of lipid type in the diet after burn injury. Annals of Surgery 204, 1-8.

Athlin L, Holmberg SB \& Hafstrom L (1991) Macrophage function and surgery. European Journal of Surgery 157, 163-170.

Atkinson S, Sieffert E \& Bihari D (1998) A prospective, randomised, double-blind, controlled clinical trial of enteral immunonutrition in the critically ill. Critical Care Medicine 26, $1164-1172$.

Barbul A (1986) Arginine biochemistry, physiology, and therapeutic implications. Journal of Parenteral and Enteral Nutrition 10, 227-238.

Barbul A, Lazarou SA, Efron DT, Wasser Krug HL \& Efron G (1990) Arginine enhances wound healing and lymphocyte immune responses in humans. Surgery 108, 331-336.

Baumann H \& Gauldie J (1994) The acute phase response. Immunology Today 15, 74-80.

Bengmark S (1998) Immunonutrition: role of biosurfactants, fibre, and probiotic bacteria. Nutrition 14, 585-594.

Bergstrom J, Furst P, Noree LO \& Vinners E (1974) Intracellular free amino acid concentration in human muscle tissue. Journal of Applied Physiology 36, 693-697.

Bone RC (1996) Toward a theory regarding the pathogenesis of the systemic inflammatory response syndrome: what we do and do not know about cytokine regulation. Critical Care Medicine 24, 163-172.

Bower RH, Cerra FB, Fershadsky B, Licari JJ, Hoyt DB, Jensen GL, VanBuren CT, Rothkopf MM, Daly JM \& Adelsberg MD (1995) Early enteral administration of a formula (IMPACT) supplemented with arginine, nucleotides, and fish oil in intensive care unit patients: results of a multicentre prospective randomised clinical trial. Critical Care Medicine 23, 436-449.

Braga M, Gianotti L, Cestari A, Vignali A, Pellega F, Dolci A \& DiCarlo V (1996a) Gut function and immune and inflammatory reponses in patients perioperatively fed with supplemented enteral formulas. Archives of Surgery 131, 1257-1264.

Braga M, Vignali A, Gianotti L, Cestari A, Profili M \& Carlo VD (1996b) Immune and nutritional effects of early enteral nutrition after major abdominal operations. European Journal of Surgery 162, 105-112.

Chang HR \& Bistrian B (1998) The role of cytokines in the catabolic consequences of infection and injury. Journal of Parenteral and Enteral Nutrition 22, 156-166.

Daly JM, Lieberman MD, Goldfine J, Shou J, Weintraub F, Rosato EF \& Lavin P (1992) Enteral nutrition with supplemented arginine, RNA, and omega three fatty acids in patients after operation: Immunologic, metabolic and clinical outcome. Surgery 112, 56-67.

Daly JM, Weintraub FN, Shou J, Rosato EF \& Lucia M (1995) Enteral nutrition during multimodality therapy in upper gastrointestinal cancer patients. Annals of Surgery 221, 327-338.

Davies MG \& O'Hagen PO (1997) Systemic inflammatory response syndrome. British Journal of Surgery 84, 920-935.

Deitch EA (1994) Bacterial translocation: the influence of dietary variables. Gut 35, Suppl. 1, S23-S27.

Edwards PD, Topping P, Kontaridis MI, Moldiver LL, Copeland EM III \& Lind DS (1997) Arginine-enhanced enteral nutrition augments the growth of a nitric oxide producing tumour. Journal of Parenteral and Enteral Nutrition 21, 215-219.
Evoy D, Lieberman MD, Fahey TJ \& Daly JM (1998) Immunonutrition: the role of arginine. Nutrition 14, 611-617.

Fish J, Sporay G, Eyer K, Jones J, Kihara T, Kennedy A, Aporan C \& Jensen GL (1997) A prospective randomized study of glutamine enriched parenteral compared with enteral feeding in postoperative patients. American Journal of Clinical Nutrition 65, 977-983.

Furst P \& Stehle P (1993) The potential use of parenteral dipeptides in clinical nutrition. Nutrition in Clinical Practice 8, 106-114.

Gaull GE \& Rassin DK (1979) Taurine and brain development: human and animal correlates. In Development and Neurobiology, pp. 461-477 [E Meisami and MAB Braziers, editors]. New York: Raven Press.

Gardiner K \& Barbul A (1993) Intestinal amino acid transport during sepsis. Journal of Parenteral and Enteral Nutrition 17, 277-283.

Gardiner KR, Gardiner RE \& Barbul A (1995) Reduced intestinal absorption of arginine during sepsis. Critical Care Medicine 23, $1227-1232$.

Giroir BP (1993) Mediators of septic shock: new approaches for interrupting the endogenous inflammatory cascade. Critical Care Medicine 21, 780-789.

Gordon RE, Shaked AA \& Solano DF (1986) Taurine protects hamster bronchioles from acute $\mathrm{NO}_{2}$ induced alterations. American Journal of Pathology 125, 585-600.

Gottschlich M, Jenkins M, Warden G, Baumer T, Havens P, Snook J \& Alexander JW (1990) Differential effects of three enteral dietary regimens on selected outcome variables in burn patients. Journal of Parenteral and Enteral Nutrition 14, 225-236.

Griffiths RD, Jones C \& Palmer TE (1997) Six month outcome of critically ill patients given glutamine-supplemented parenteral nutrition. Nutrition 13, 295-302.

Hall JC, Heel K \& McCauley R (1996) Glutamine. British Journal of Surgery 83, 305-312.

Hoffman WD \& Natanson C (1993) Endotoxin in septic shock. Anesthesia and Analgesia 77, 613-624.

Hong RW, Helton WS, Rounds JD \& Wilmore DW (1990) Glutamine supplemented TPN preserves hepatic glutathione and improves survival following chemotherapy. Surgical Forum 41, 9-11.

Hong RW, Rounds DJ, Helton WS, Robinson MK \& Wilmore DW (1992) Glutamine preserves liver glutathione after lethal hepatic injury. Annals of Surgery 215, 114-119.

Houdijk AP, Rjinsburger ER, Jansen J, Wesdorp J, Weiss JK, McCarnish MA, Teerlink T, Meuwissen SG, Haarman HJ, Thijs LG \& van Leeuwen PA (1998) Randomised trial of glutamineenriched enteral nutrition on infectious morbidity in patients with multiple trauma. Lancet 352, 772-776.

Huxtable RH (1992) Physiological actions of taurine. Physiological Reviews 72, 101-163.

Kohn CL \& Keithly JK (1989) Enteral nutrition: potential complications and patient monitoring. Nursing Clinics of North America 24, 339-353.

Lacey JM \& Wilmore DW (1990) Is glutamine a conditionally essential amino acid? Nutrition Reviews 48, 297-309.

Lieberman MD, Nishioka K, Redmond BP \& Daly JM (1992) Enhancement of interleukin-immunotherapy with L-arginine. Annals of Surgery 215, 157-165.

MacBurney M, Young LS, Ziegler TR \& Wilmore DW (1994) A cost-evaluation of glutamine-supplemented parenteral nutrition following bone marrow transplantation. Journal of the American Dietetic Association 94, 1263-1266.

McLoughlin DM, Stapleton PP \& Bloomfield FJ (1991) Influence of taurine and a substituted taurine on the respiratory burst pathway in the inflammatory response. Biochemical Society Transactions 19, 73-78. 
Masuda M, Horiksaka K \& Koeda T (1986) Effect of taurine on neutrophil function in hyperlipidaemic rats. Japanese Journal of Pharmacology 40, 478-480.

Morlion BJ, Stehle P, Wachtler P, Siedhoff HP, Koller M, Konig M, Furst P \& Puchstein C (1998) Total parenteral nutrition with glutamine dipeptide after abdominal surgery: a randomised double blind, controlled study. Annals of Surgery 227, 302-308.

O'Flaherty L, Stapleton PP, Redmond HP \& Bouchier-Hayes DJ (1997) Dexamethasone and lipopolysaccharide regulation of taurine transport in Caco-2 cells. Journal of Surgical Research 69, 331-336.

O'Flaherty L, Stapleton PP, Redmond HP \& Bouchier-Hayes DJ (1998) Taurine modulates immune response and reduces bacterial translocation and gut atrophy in a murine model of sepsis. Proceedings of the Nutrition Society 57, 143A.

O'Riordán MG, De Beaux DE \& Fearon KCH (1996) Effect of glutamine on immune function in the surgical patient. Nutrition 12, S82-S84.

Pasantes-Morales H, Wright CE \& Gaull GE (1985) Taurine protection of lymphoblastoid cells from iron-ascorbate induced damage. Biochemical Pharmacology 34, 2205-2207.

Park KG, Hey SD, Blessing K, Kelly P, McNurlan MA, Emermion O \& Garlick PJ (1992) Stimulation of human breast cancers by dietary arginine. Clinical Science 82, 413-417.

Reynolds JV, Daly JM \& Zhang S (1998) Immunomodulatory mechanisms of arginine. Surgery 194, 142-151.

Senkal M, Kemen M, Homann HH, Eickhoff U, Baier J \& Zumtobel V (1995) Modulation of postoperative immune responses by enteral nutrition by a diet enriched with arginine, RNA and omega three fatty acids in patients with upper gastrointestinal cancer. European Journal of Surgery 161, $115-122$.

Senkal M, Mumme A, Eickhoff U, Geier B, Spath G, Wulfert D, Joosten U, Frei A \& Kemen M (1997) Early postoperative enteral immunonutrition: clinical outcome and cost-comparison analysis in surgical patients. Critical Care Medicine 25, 1489-1496.

Slade MS, Simmons RL, Yumis E \& Greenberg LJ (1975) Immunodepression after major surgery in normal patients. Surgery 78, 363-372.
Souba WW (1993) Intestinal glutamine metabolism and nutrition. Journal of Nutritional Biochemistry 4, 2-8.

Stapleton PP, Redmond HP \& Bouchier-Hayes DJ (1998) Myeloperoxidase (MPO) may mediate neutrophil adherance to the endothelium through upregulation of CD11b expression-an effect down regulated by taurine. Advances in Experimental and Medical Biology 442, 183-192.

Utoh J, Yamamoto T, Utsonomiya T, Kambara T, Goto H \& Miyauchi Y (1988) Effect of surgery on neutrophil functions, superoxide and leukotriene production. British Journal of Surgery 75, 682-685.

Wang XD, Andersson R, Soltesz V, Wang WQ, Ar'Rajab A \& Bengmark S (1994) Phospholipids prevent enteric bacterial translocation in the early stage of experimental acute liver failure in the rat. Scandinavian Journal of Gastroenterology $\mathbf{2 9}$, 1117-1121.

Watson RWG, Redmond HP \& Bouchier-Hayes D (1994) Taurine upregulates antimicrobial function of human inflammatory cells. Surgical Forum 45, 679-681.

Weimann A, Bastian L, Bischoff WE, Grotz M, Lotz J, Trautwein C, Tusch G, Schlitt HJ \& Regel G (1998) Influence of arginine, omega-3 fatty acids and nucleotide supplemented enteral support on systemic inflammatory response syndrome on multiple organ failure in patients after severe trauma. Nutrition 14, 165-172.

Wilmore D \& Shabert JK (1998) Role of glutamine in immunologic responses. Nutrition 14, 618-626.

Yamaguchi S, Miyazaki Y, Oka S \& Yano I (1994) Stimulatory effect of gangliosides phagosome-lysosome fusion, and intracellular signal transduction system by human polymorphonuclear leukocytes. Glycoconjugate Journal 14, 707-714.

Yeatman TJ, Risley GL \& Brunson ME (1991) Depletion of dietary arginine inhibits growth of metastatic tumor. Archives of Surgery 126, 1376-1381.

Zaporozhets TS, Besednova NN, Liamkin GP, Leonko IN \& Popov AM (1991) Immunodulating properties of pectin from sea grass zostera. Antibiotiki i Khimioterapira 36, 31-34.

Ziegler TR, Bye RI, Persiner RI, Young LS, Antin JH \& Wilmore DW (1998) Effects of glutamine supplementation: a pilot study. American Journal of Medical Science 315, 4-10. 